\title{
APP717 and Alzheimer's disease in Italy
}

Sir - A possible pathogenetic mutation in the amyloid precursor protein (APP) gene causing a Val to Ile change at codon 717 has been reported in seven families with early onset familial Alzheimer's disease (EOFAD), four of British origin and three Japanese $\mathrm{e}^{1-5}$. It has been suggested

$a$
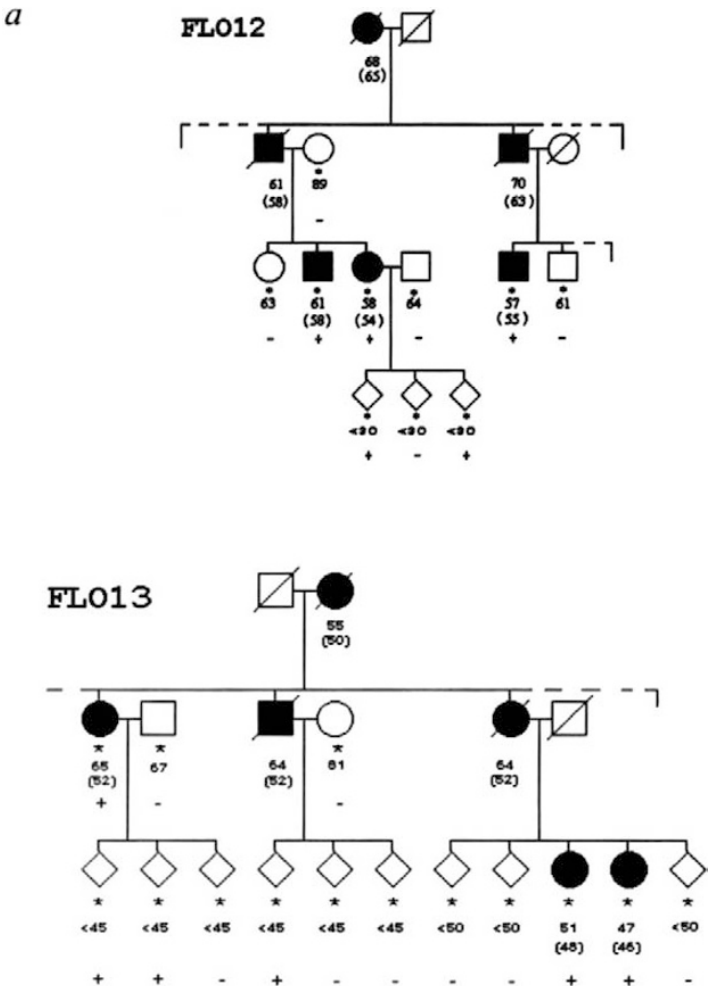

b

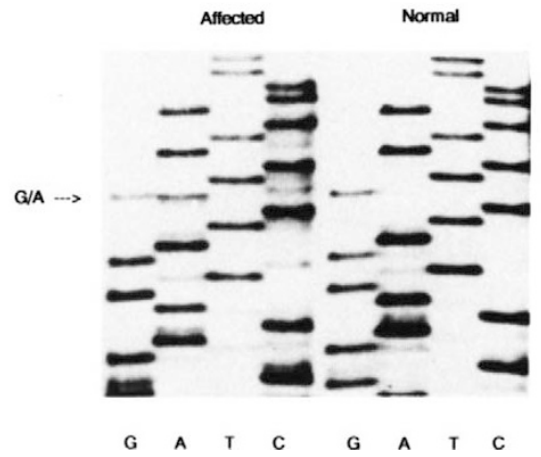

Fig. 1 a, Simplified pedigrees of FLO12 and FLO13. Mean age-atonset of cognitive symptoms ( \pm sd) is $58 \pm 4$ and $50 \pm 3$ years, respectively. Clinical diagnosis fulfilled the NIH-NINCDS criteria and was confirmed at autopsy. Filled symbols represent affected individuals. Age is shown beneath the symbols. Age at onset is shown in parenthesis. " indicates individuals studied by PCR and DNA sequencing. +/- indicate presence or absence of the APP .Val717lle mutation. $b$, Autoradiograph of sequencing gel from a portion of APP exon 17 in an AD patient showing a single base pair change and in a normal relative. Arrow indicates the position of the $\mathrm{G}$ to $\mathrm{A}$ transition. A part of exon 17 of $A P P$ was amplified by PCR using the following exonic primers generating a DNA fragment of 147 bp: (i) 5' GAA TTC CTC CAC CAC ACC ATG ATG AAT-3'; (ii) 5' AAG CTI GTG TTC TTI GCA GAA GAT GTG-3'. PCR product sequencing was performed with Sequenase version 2.0 using the same primers. The presence of the mutation was confirmed after sequencing of the opposite strand. that the clustering of such cases on two island nations argues for the existence of a common founder in each country ${ }^{6}$.

We have studied six well characterized, autopsy proven early onset ( $<60$ years) Italian FAD families not linked by genealogyby polymerase chain reaction (PCR) amplification of APP exon 17 and direct sequencing. Two families, FLO12 and FLO13, had the Val717Ile mutation segregating with the disease (Fig. 1); the mutation was found in all affected as well as in two members of FLO12 and three members of FLO13 whose age is below the age of onset. No persons older than the age of onset and without clinical manifestations of the disease showed the mutation. The mutation was not found in three patients with late-onset sporadic $\mathrm{AD}$ and in thirty unrelated apparently normal controls.

This finding suggests that: (i) the APP Val717Ile mutation is not confined to islander FAD families which may share a common founder. It is unlikely that Japanese, British and Italian kindreds all have a common origin. (ii) The frequency of the Val717lle mutation is probably higher than generally considered, at least in kindreds of Italian origin. APP717 mutation was found in 33\% of our families. This result is consistent with previous studies reporting Val717Ile in 2 out of $16(12.5 \%)$ EOFAD families ${ }^{1}, 2$ out of $3(66 \%)^{2}, 1$ out of $6(15 \%)^{3}$, and 1 out of $21(5 \%)^{4}$. The overall incidence of these positive reports is about $17 \%$. Furthermore at least two other mutations (Val to Phe and Val to Gly) at codon 717 have been reported ${ }^{7,8}$. However other groups $^{9-14}$ have published negative results in large series of early and late onset families and sporadic cases. Such differences may reflect variability in gene frequency in different populations. However the reliability of techniques used by different groups is not known. At least one false positive APP717 mutated family has been published ${ }^{15-16}$. Moreover, FLO13, one of our positive families, was also recently reported to be without any mutation in exons 16 and 17 (ref. 12). The true frequency of APP mutation will be missed if false negative or false positive are included. The entire APP gene has been studied only in a few affected EOFAD patients ${ }^{4,12}$. Linkage analysis with APP markers may help in the screening of FAD families. The frequency of APP gene mutations is of crucial relevance for the understanding of the etiopathogenetic role of amyloid in Alzheimer's disease, particularly after the recent observation that some families have a mutated gene on chromosome 14 (refs 17-20).

Among the few Italian kindreds with EOFAD studied so far two have provided independent evidence of linkage to chromosome 14 (ref. 18), supporting the view of genetic heterogeneity of $\mathrm{AD}$.

Sandro Sorbi

Benedetta Nacmias

Paolo Forleo

Silvia Piacentini

Luigi Amaducci

Department of Neurological and

Psychiatric Sciences,

University of Florence,

Viale Morgagni 85, Florence, Italy

Leandro Provinciali

Department of Neurology,

University of Ancona,

Ospedale Torrette, Ancona, Italy

\section{References}

1. Goate, A.M. et al. Nature 349, 704-706 (1991).

2. Naruse, S. et al. Lancet 337, 978-979 (1991).

3. Yoshioka, K. et al. Biochem. Biophys. Res. Commun. 178, 1141-1146 (1991).

4. Fidani, L. et al. Hum. molec. Genet. 1, 165-168 (1992).

5. Karlinsky, H. etal. Neurology 42, 1445-1453(1992).

6. St George-Hyslop, P.H. et al. In Heterogeneity of Alzheimer's disoase (eds Boelier, F. et al.) 88-95 (Springer, Berlin, 1992).

7. Murrell, J., Farlow, M., Ghetti, B. \& Benson, M. Science 254, 97-99 (1991).

8. Chartier-Harlin, M.C. et al. Nature $353,844-846$ (1991).

9. Chartier-Harlin, M.C., Crawford, F., Hamandi, K., Mullan, M. \& Goate, A. Neurosci. Lett. 129, 134135 (1991).

10. Schellenberg, G.D. et al. Am. J. hum. Genet. 49, $511-517$ (1991).

11. Van Dujin, C.M. et al. Lancet 337, 978 (1991).

12. Tanzi, R. et al. Am. J. hum. Genet. 51, 273-282 (1992).

13. Karnino, K. et al. Am. J. hum. Genet. 51, 998-1014 (1992).

14. Crawford, F. et al. Neurosci. Lett. 133, 1-3(1991).

15. Lucotte, G., Berriche, S. \& David, F. Nature 351, 530 (1991).

16. Crawford, F. et al. Nature 356, 390 (1992)

17. Schellenberg, G.D. et al. Science 258, 668-671 (1992).

18. St George-Hyslop, P.H. et al. Nature Genet. 2, 330-334 (1992).

19. Van Broeckhoven, C. et al. Nature Genet. 2, 335339 (1992).

20. Mullan, M. et al. Nature Genet. 2, 340-342 (1992).

\section{Acknowledgements}

We thank L. Papi, L. De Vitis and C. Ballerini for technical assistance and M. Goedert and M.G. Spillantini for providing the sequences of exon 17 primers. Supported by Consiglio

Nazionale delle Ricerche (C.N.R., Rome, Italy).

Correspondence should be addressed to S.S. 